\title{
High-resolution nanopatterning by achromatic spatial frequency multiplication with electroplated grating structures
}

\author{
Li Wang, Bernd Terhalle, Mohamad Hojeij, and Vitaliy A. Guzenko \\ Laboratory for Micro- and Nanotechnology, Paul Scherrer Institute, CH-5232 Villigen PSI, Switzerland \\ Yasin Ekinci ${ }^{\mathrm{a})}$ \\ Laboratory for Micro- and Nanotechnology, Paul Scherrer Institute, CH-5232 Villigen PSI, Switzerland \\ and Department of Materials, Laboratory of Metal Physics and Technology, ETH Zürich, CH-8093 Zürich, \\ Switzerland
}

(Received 6 January 2012; accepted 6 March 2012; published 28 March 2012)

\begin{abstract}
The authors demonstrate generation of high-resolution nanostructures using achromatic spatial frequency multiplication in the extreme ultraviolet wavelength region. The technique based on the achromatic Talbot effect is used for periodic transmission gratings under wideband illumination, enabling one- and two-dimensional nanopatterns with sub- $20 \mathrm{~nm}$ feature sizes. The transmission masks with desired properties are fabricated with electron-beam lithography followed by electroplating of gold. Features sizes down to $12 \mathrm{~nm}$ are obtained. The presented technique provides highthroughput and large-area nanopatterning with great flexibility in tuning pattern parameters such as linewidth and dot size. (C) 2012 American Vacuum Society. [http://dx.doi.org/10.1116/1.3697753]
\end{abstract}

\section{INTRODUCTION}

Nanolithography is the major driving force behind the rapid progress in nanotechnology. High-resolution nanopatterning, in particular with feature sizes in the sub-20 nm regime, remains a challenge for most of the lithographic techniques and continues to emerge as an important research area in modern nanotechnology with substantial impact on various fields ranging from industrial manufacturing to academic research. Currently, various methods of top-down and bottom-up approaches have been pursued and demonstrated. While the state-of-the-art in semiconductor manufacturing is based on optical double-patterning methods and immersion lithography at a wavelength of $\lambda=193 \mathrm{~nm}$, other techniques, such as nanoimprint lithography, helium beam patterning, electron-beam lithography (EBL), and self-assembly methods, have also been proven to be capable of high-resolution patterning. ${ }^{1-3}$ In terms of future technologies for highvolume manufacturing, particular interest is currently focused on extreme-ultraviolet (EUV) lithography. ${ }^{4-6}$ In parallel with projection optical systems developed for industrial applications, extreme-ultraviolet interference lithography (EUV-IL) is used as a powerful tool for both scientific and industrial research, ${ }^{7,8}$ which combines the advantages of a parallel fabrication process with high resolution and high throughput. Dedicated EUV-IL tools have been set up using synchrotron, ${ }^{9}$ laser sources, ${ }^{10}$ and plasma sources. ${ }^{11}$ The EUV-IL method plays an important role in the development of chemically amplified resists for EUVL by providing highresolution exposures before high-NA projection tools become available. ${ }^{12,13}$ Recently, it has been successfully employed for the generation of sub-10 nm structures, which currently marks the record in photon-based lithography. ${ }^{14}$

In addition to the most commonly used method of two- or multiple-beam interference lithography employing transmis-

$\overline{{ }^{a} \text { Electronic mail: yasin.ekinci@psi.ch }}$ sion diffraction gratings, ${ }^{8}$ the so-called achromatic spatial frequency multiplication (ASFM) has also been demonstrated with EUV light and implemented for the fabrication of $1 \mathrm{D}$ and $2 \mathrm{D}$ periodic nanostructures with periods of several hundred nanometers. ${ }^{15}$ The ASFM technique is based on the achromatic Talbot effect, ${ }^{16}$ which results in a stationary areal image behind a grating illuminated with a source of limited coherence. This technique provides several advantages over multiple-beam interference. Since in this technique the pattern is formed by a single grating, it enables patterning with the full area of the illuminated spot, whereas in multiplebeam interference, the patterned area is significantly smaller than the illuminated area. Moreover, in ASFM technique all the transmitted light contributes to the image formation, whereas in multiple-beam interference only a small fraction, e.g., first diffraction orders, are used. These two advantages are of great importance for tabletop EUV-IL setups where beam size and flux are the main bottleneck of plasma and laser sources. In this paper, we demonstrate the suitability of ASFM for the generation of 1D and 2D high-resolution periodic nanostructures with feature sizes in the sub-20 nm range. We describe the fabrication of high-quality transmission masks using EBL followed by electroplating of $\mathrm{Au}$, which is needed for the formation of high-contrast areal image.

\section{THEORY AND SIMULATION}

In the ASFM method the transmission function of the grating has a substantial influence on the areal image formed. In order to illustrate this effect, we briefly discuss the basic principle of the ASFM method as shown in Fig. 1. When a periodic structure with period $p$ is illuminated with coherent light of wavelength $\lambda$, self-images of the structure are formed at multiples of the so-called Talbot distance $z_{T}=p^{2} / \lambda$ with consecutive self-images being laterally shifted by half a period. ${ }^{16}$ For wideband illumination with a 
spectral width of $\Delta \lambda$, contributions to the self-image arising from different wavelengths overlap and give rise to a stationary intensity pattern beyond a certain distance of $z_{a}=2 p^{2} / \Delta \lambda^{15}$ The resulting intensity pattern is invariant in $z$ and has twice the spatial frequency of the illuminated parent grating. This is illustrated in Fig. 1 for rectangular and sinusoidal grating profiles with period $p=200 \mathrm{~nm}$. The image formation was calculated numerically using an angular spectrum propagation method. The gratings were assumed to be infinitely thin, i.e., the propagation within the grating itself was neglected. Plane wave illumination at a wavelength of $\lambda=13.5 \mathrm{~nm}$ at normal incidence was assumed and the relative bandwidth of a Gaussian spectral distribution was set to $\Delta \lambda / \lambda=4 \%$. The contributions of different wavelengths to the final intensity distribution were calculated separately and added according to their spectral weight. Using these parameters, the approximate distance for the formation of a stationary intensity distribution can be calculated to $z_{a} \approx 148 \mu \mathrm{m}$ and Figs. 1(c) and 1(d) clearly confirm the formation of stationary images for $z>z_{a}$. It should be noted that in case of a monochromatic illumination, a similar result can be achieved by integrating the resulting intensity distribution in the propagation direction by moving the sample during the exposure. ${ }^{17}$

As an intuitive approximation, the areal image formed in the ASFM technique can be understood as the autocorrelation function of the grating transmission profile. Consequently, the rectangular grating transmission function leads to a triangular image profile [Fig. 1(e)] while the image resulting from a sinusoidal transmission function remains sinusoidal with twice the frequency of the grating structure [Fig. 1(f)]. The areal image formation in 1D and 2D cases is illustrated in Fig. 2. In case of 2D gratings, such as hole [Fig. 2(d)] and pillar arrays [Fig. 2(g)], the calculated areal images [Figs. 2(e) and 2(h)] at propagation distance $z$ of $3000 \mu \mathrm{m}$ show sharp peaks on a $45^{\circ}$ rotated square grid with a period equal to the mask period divided by $\sqrt{2}$. These two calculated areal images from 2D gratings of hole and pillar arrays present similar features, because of the autocorrelation function of the grating transmission profile. The areal
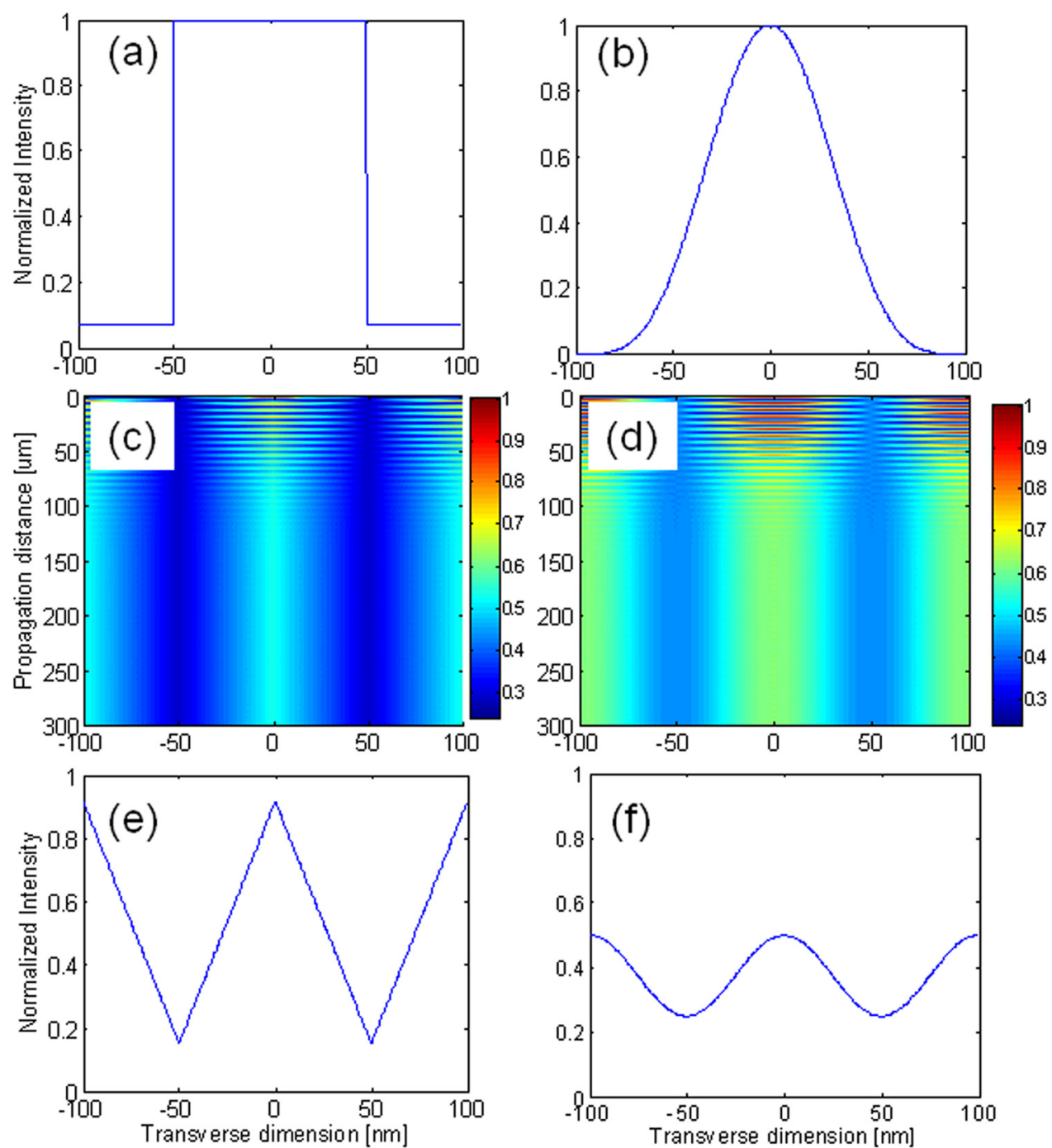

FIG. 1. (Color online) Numerical simulations of areal image formation with rectangular (left column) and sinusoidal (right column) grating profiles. Transmission functions of masks with (a) rectangular and (b) sinusoidal grating profiles. (c), (d) Intensity profiles of areal images formed behind the grating illuminated with spatially coherent light of $5 \%$ bandwidth. (e), (f) One-dimensional profiles of the areal image obtained at $z=300 \mu \mathrm{m}$. 
image profiles of the 2D gratings with rectangular cross sections also show triangular shaped features [Figs. 2(f) and 2(i)]. The small peaks in the areal image profiles resulting from higher diffraction orders of the grating have much lower intensity than the sharp peaks, and thus they can be easily avoided in exposures at low doses. In terms of nanolithography, the triangular profile is clearly advantageous since the contrast of the areal image, i.e., its slope, is constant over the whole period. In multiple-beam interference scheme and in ASFM using masks with sinusoidal transmission profile, the areal image is sinusoidal and the contrast diminishes at extreme duty cycles. Consequently, the use of an ASFM mask with sharp rectangular profiles offers the possibility to achieve smaller feature sizes by adjusting the exposure dose to the tips of the individual triangles and allows for greater flexibility in varying linewidth and duty cycle than the sinusoidal profile. The challenge lies in the fabrication of high-resolution masks with rectangular transmission function as closely as possible to the ideal case. To achieve this, we developed a fabrication process in which the grating formation is based on electroplating, which enables realization of steeper grating profiles than other methods such as reactive ion etching and lift-off processes.

A disadvantage of the ASFM technique is the significant background in the areal image modulation. The relative value of the background strongly depends on the grating parameters. We performed extensive calculations in order to determine the optimal geometrical parameters of the mask
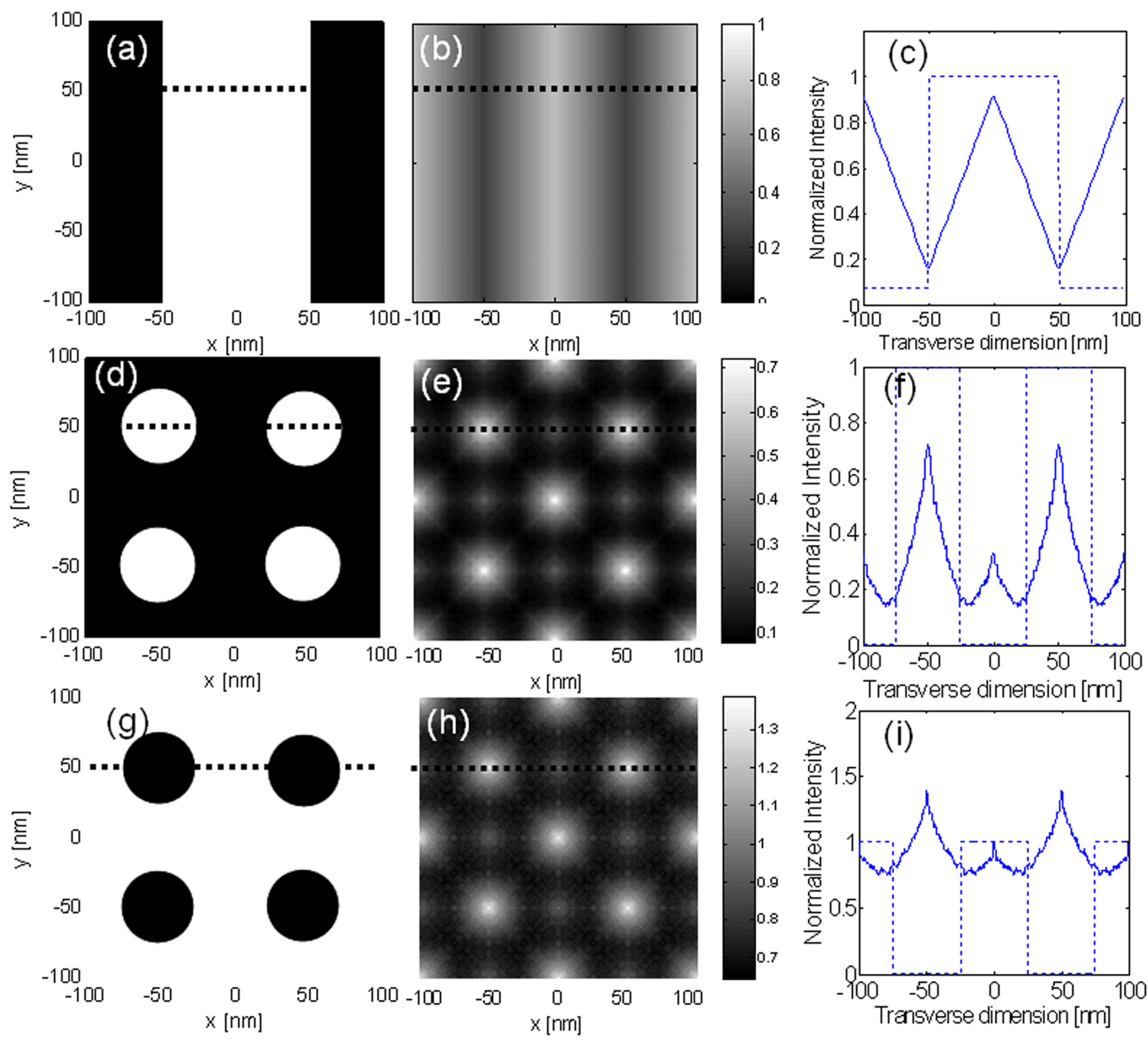

FIG. 2. (Color online) Transmission of the one- (a) and two-dimensional hole (d) and pillar (g) masks showing one period and four unit cells of the periodic structures, respectively. In (a) the period and the linewidth are 200 and $100 \mathrm{~nm}$, respectively. In (d) and (g) the period is $100 \mathrm{~nm}$ and hole or pillar diameter is $50 \mathrm{~nm}$. (b), (e), (h) Calculated areal images at propagation distance of $z=300 \mu \mathrm{m}$ (b) and $3000 \mu \mathrm{m}$ (e), (h). (c), (f), (i) Profiles of the images in (a), (b); (d), (e); and (g), (h) along the black dashed lines, where the dashed and solid lines represent mask transmission and areal image intensity, respectively. 
gratings, such as electroplated metal $(\mathrm{Au})$ thickness and duty cycle, within the feasible range of parameters in terms of nanofabrication. Figure 3 shows the dependence of the visibility on grating parameters. The visibility is defined as $\left(I_{\max }-I_{\min }\right) /\left(I_{\max }+I_{\min }\right)$, where the intensities are the maximum and minimum intensities extracted from the areal image intensity profiles, such as in Figs. 2(c), 2(f), and 2(i), as a function of $1 \mathrm{D}$ grating duty cycles, and $2 \mathrm{D}$ grating pillar/hole diameters. The calculations are performed for two different thicknesses of 55 and $100 \mathrm{~nm}$. The former value is expected to provide the highest visibility, since the maximum first-order diffraction efficiency is achieved by an optimal phase shift of $\pi$, which is obtained at a grating thickness of $55 \mathrm{~nm}$ according the reported optical properties of $\mathrm{Au}$ at $13.5 \mathrm{~nm}$ wavelength. ${ }^{18}$ However, in our preliminary experi-
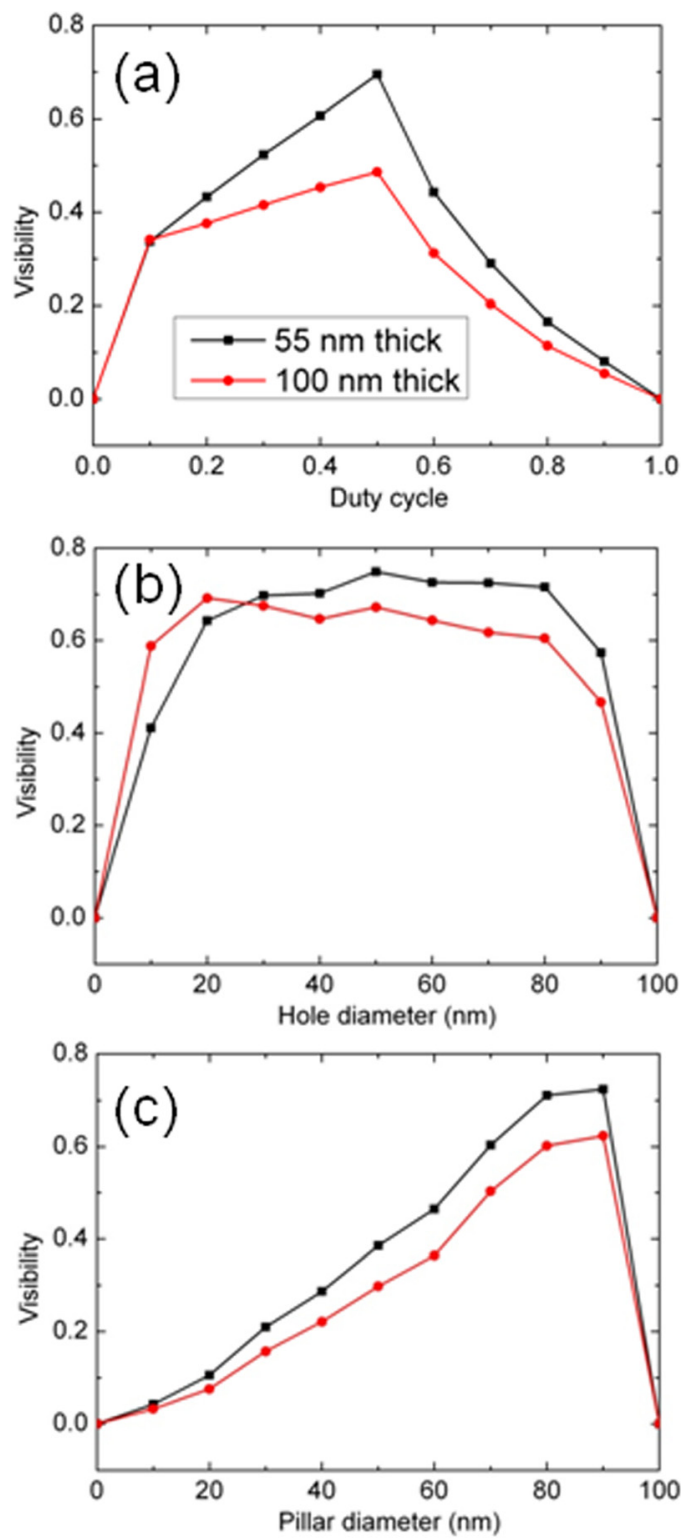

FIG. 3. (Color online) Visibility $\left[\left(I_{\max }-I_{\min }\right) /\left(I_{\max }+I_{\min }\right)\right]$ of the stationary image from the $1 \mathrm{D}$ (a) and $2 \mathrm{D}(\mathrm{b})$, (c) grating masks as a function of grating duty cycle or feature diameters in two different grating metal thicknesses. ments we observed that the rough surface of electroplated $\mathrm{Au}$ thin film induces incoherent scattering and therefore reduces the image quality and uniformity. ${ }^{19}$ On the other hand, for a thickness of $100 \mathrm{~nm}$, the transmission through the Au layer low and thereby the effect of surface roughness is negligible. The periods for $1 \mathrm{D}$ and $2 \mathrm{D}$ gratings in the calculation are 200 and $100 \mathrm{~nm}$, respectively. Figure 3(a) shows the dependence of the visibility on the grating duty cycle, which reaches its maximum at a duty cycle of 0.5 with $\mathrm{Au}$ thickness of $55 \mathrm{~nm}$, almost two times more than that with $\mathrm{Au}$ thickness of $100 \mathrm{~nm}$. For the case of 2D hole or pillar gratings, despite the similarity of areal images obtained in both cases, the dependence of the visibility on hole and pillar diameters is different. Generally, pillar gratings provide lower visibility than that of the hole grating. This is due to the relatively large portion of nondiffracted light passing through the open areas in pillar gratings, which contributes to the background in the areal image. The visibility of hole gratings is relatively independent of the hole diameter (except extreme cases) and Au thickness. Based on our calculations and considerations of feasibility in terms of nanofabrication, we decided to fabricate two ASFM masks with a period $200 \mathrm{~nm}$, a duty cycle of 0.5 , and an Au thickness of $55 \mathrm{~nm}$ for 1D mask, and with a period of $100 \mathrm{~nm}$, a hole diameter of $50 \mathrm{~nm}$, and an Au thickness of $100 \mathrm{~nm}$ for 2D mask.

\section{EXPERIMENTAL RESULTS AND DISCUSSION}

The process flows for the fabrication of these two different EUV transmission masks are shown in Fig. 4 schematically.

(a)

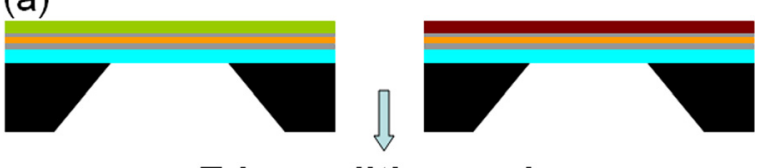

(b)

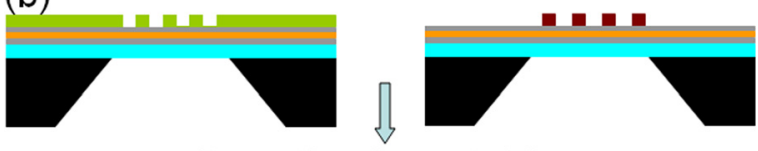

Reactive ion etching

(c)

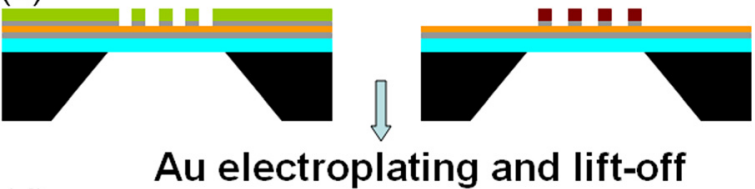

(d)

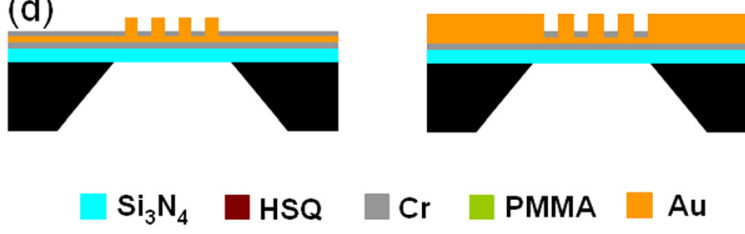

FIG. 4. (Color online) Schematic illustration of the mask fabrication process with HSQ (left column) and PMMA (right column) for one- and twodimensional gratings, respectively. (a) $100 \mathrm{~nm} \mathrm{Si} 3 \mathrm{~N}_{4}$ membrane with thermally evaporated $\mathrm{Cr} / \mathrm{Au} / \mathrm{Cr}(2 \mathrm{~nm} / 5 \mathrm{~nm} / 2 \mathrm{~nm})$ tri-layer and spin-coated resist layer; (b) electron-beam lithography; (c) reactive-ion etching; (d) Au electroplating and resist removal. 

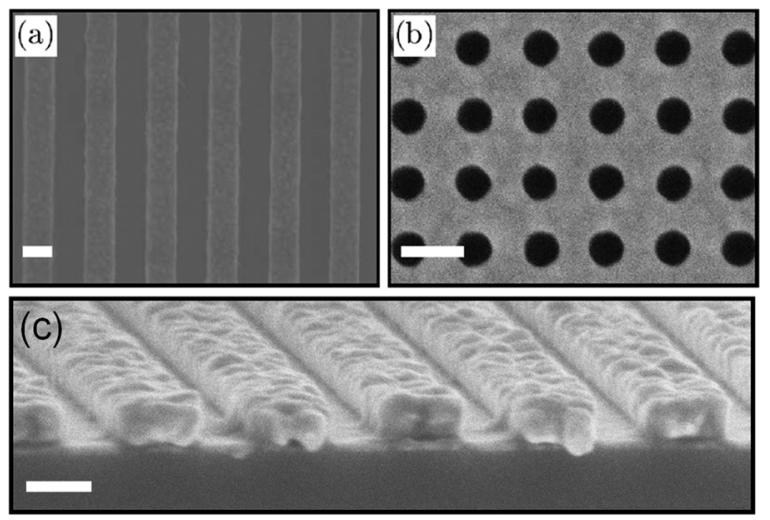

FIG. 5. SEM images of the fabricated masks. (a) Top-down view and (c) $80^{\circ}$-tilted view of the one-dimensional grating with period $p=200 \mathrm{~nm}$; (b) two-dimensional grating with period $p=100 \mathrm{~nm}$. Scale bars correspond to $100 \mathrm{~nm}$.

Both masks were fabricated on a 100 -nm-thick $\mathrm{Si}_{3} \mathrm{~N}_{4}$ membrane with a thermally evaporated $\mathrm{Cr} / \mathrm{Au} / \mathrm{Cr}$ multilayer having a thickness of $2 / 5 / 2 \mathrm{~nm}$, respectively. For the first mask, a 1D grating with a period of $200 \mathrm{~nm}$, the membrane was coated with a 220-nm-thick layer of poly(methyl methacrylate) (PMMA) of 950000 molecular weight and the grating structures were exposed with an EBL tool (Vistec EBPG5000+) at $100 \mathrm{keV}$ electron energy. Following the exposure, the samples were developed in a solution made of $7: 3$ isopropyl alcohol (IPA) and water for $15 \mathrm{~s}$. A chlorine dry etching process was then used to etch through the top Cr layer in order to facilitate the subsequent Au electroplating of the grating bars to a thickness of approximately $55 \mathrm{~nm}$. The electroplating process of $\mathrm{Au}$ was optimized in order to obtain low-stress Au films, because of the limited mechanical stability of the membranes. Finally, the fabrication was completed by the removal of residual PMMA with an oxygen plasma. Figures 5(a) and 5(c) show scanning electron microscopy (SEM) images of the electroplated $\mathrm{Au}$ grating, indicating rectangular-shaped cross-sectional profile. As a second mask, we fabricated a 2D hole array with a period of $100 \mathrm{~nm}$ [cf. Fig. 5(b)]. In this case, the $\mathrm{Si}_{3} \mathrm{~N}_{4}$ membrane was spin-coated with negative tone hydrogen silsesquioxane (HSQ) resist (FOX 14, Dow Corning) of $100 \mathrm{~nm}$ thickness. The gratings were written by EBL and subsequently developed in a $1 \%$ aqueous solution of $\mathrm{NaOH}$ for $4 \mathrm{~min}$, resulting in a square array of HSQ pillars of approximately $50 \mathrm{~nm}$ in diameter. Similar to the previous case, we used a chlorine plasma etching process to remove the top $\mathrm{Cr}$ layer followed by $\mathrm{Au}-$ electroplating of the areas in between the pillars. The final hole grating was eventually obtained by removal of HSQ in buffered oxide etcher. An SEM image of the obtained mask is shown in Fig. 5(b).

The EUV exposures were performed at the XIL-II beamline at the Swiss Light Source (SLS) with a spatially coherent EUV light of $13.5 \mathrm{~nm}$ wavelength with a bandwidth of $\Delta \lambda / \lambda=4 \%$. The patterns were recorded on Si wafers coated with HSQ resist (FOX XR1541, Dow Corning) at a spinning speed of $5000 \mathrm{rpm}$ for $45 \mathrm{~s}$, resulting in a thickness of $35 \mathrm{~nm}$. The gap between mask and sample was set to $1 \mathrm{~mm}$

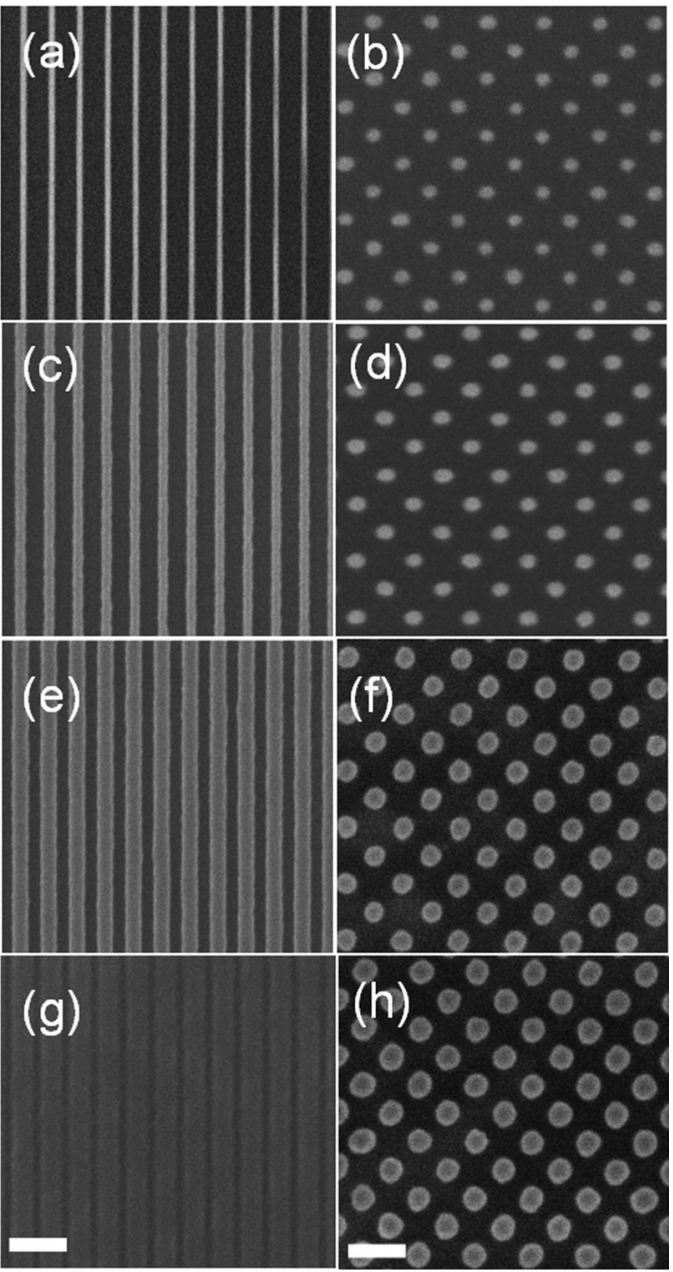

FIG. 6. SEM images of the experimentally obtained patterns exposed in HSQ resist with low dose (a), (b); medium dose (c)-(f); and high dose (g), (h). Scale bars correspond to $200 \mathrm{~nm}$ (a), (c), (e), (g) and $100 \mathrm{~nm}$ (b), (d), (f), (h), respectively.

and the exposure dose was varied between 20 and 200 $\mathrm{mJ} / \mathrm{cm}^{2}$. Following the exposure, the samples were developed in a $25 \%$ aqueous solution of tetramethylammonium hydroxide (TMAH) for $60 \mathrm{~s}$. The results are summarized in Fig. 6. Using the two different grating masks, we obtained linewidths down to $12 \mathrm{~nm}$ for the $1 \mathrm{D}$ pattern of $100 \mathrm{~nm}$ period [Fig. 6(a)] and dot sizes as small as $15 \mathrm{~nm}$ for the 2D pattern of $70 \mathrm{~nm}$ periods [Fig. 6(b)], respectively. We should note here, the aspect ratios of up to 3 are obtained with such small nanostructures. Sub-10 nm lines and dots may be possible with extensive process optimization. By varying the doses, we have been able to change the duty cycle of the exposed pattern between 0.1 and 0.9 for the $1 \mathrm{D}$ patterns and between 0.2 and 0.7 for the 2D structures, respectively. Figures 6(c)-6(h) show SEM images of patterns with duty cycles of approximately $0.3-0.9$ and $0.3-0.7$ for $1 \mathrm{D}$ and $2 \mathrm{D}$ structures, respectively.

\section{CONCLUSIONS}

In conclusion, we have demonstrated high-resolution nanopatterning using the ASFM technique. We designed 
efficient 1D and 2D grating masks by numerical simulations considering the feasible nanofabrication processes. For the fabrication of high-quality periodic transmission gratings, we have developed and implemented an $\mathrm{Au}$ electroplating process, which enables a close approximation of the ideal rectangular mask transmission function. The resulting stationary triangular image profile offers great flexibility in tuning pattern linewidth and dot size, owing to the high contrast of the areal image at extreme duty cycles for both 1D and 2D periodic nanostructures. We have demonstrated feature sizes down to $12 \mathrm{~nm}$. Further optimization of the mask fabrication and resist processing will be explored in order to achieve sub-10 nm features. It is well known that HSQ is a high-resolution resist for both EUV-IL (Refs. 8, 14, and 20) and electron-beam lithography. ${ }^{21}$ Moreover, secondary electron blur of EUV light is estimated as a few nanometers. ${ }^{22}$ Therefore, we believe that achieving smaller features should be possible with this method owing to the sharp tip of the areal image profile. However, factors such as the background of the areal image may also limit the ultimate resolution. Optimization of the HSQ processing for the specific areal image conditions can increase the resolution. For the current study, we cannot rule out the other potential limiting factors such as the quality of the gratings and the stability of the interferometer. In addition to its potential for ultra-high-resolution patterning, this technique is a high throughput and largeare method, since it takes full advantage of the beam size and diffracted orders of the grating. Thanks to these advantages, this technique is particularly suitable for EUV interference lithography with most available EUV sources, such as synchrotron, laser, and plasma sources, and therefore can be effectively employed for highresolution nanopatterning with high throughput over large areas.

\section{ACKNOWLEDGMENTS}

The authors thank Michaela Vockenhuber and Markus Kropf for technical support. This project was funded by the NanoArgovia program of Swiss Nanoscience Institute Basel (SNI). Part of this work was performed at the Swiss Light Source (SLS), Paul Scherrer Institute, 5232 Villigen PSI, Switzerland.

${ }^{1}$ V. Sidorkin, E. van Veldhoven, E. van der Drift, P. Alkemade, H. Salemink, and D. Maas, J. Vac. Sci. Technol. B 27, L18 (2009).

${ }^{2}$ Y. S. Jung, J. B. Chang, E. Verploegen, K. K. Berggren, and C. A. Ross, Nano Lett. 10, 1000 (2010).

${ }^{3}$ A. Tavakkoli, K. G. S. N. Piramanayagam, M. Ranjbar, R. Sbiaa, and T. C. Chong, J. Vac. Sci. Technol. B 29, 011035 (2011).

${ }^{4}$ International Technology Roadmap for Semiconductors, http://www.itrs.net/.

${ }^{5}$ C. Wagner and N. Harned, Nature Photon. 4, 24 (2010).

${ }^{6}$ G. Tallents, E. Wagenaars, and G. Per, Nature Photon. 4, 809 (2010).

${ }^{7}$ H. H. Solak, J. Phys. D: Appl. Phys. 39, R171 (2006).

${ }^{8}$ V. Auzelyte et al., J. Micro/Nanolith. MEMS MOEMS 8, 021204 (2009).

${ }^{9}$ J. Wallace, Y. C. Cheng, A. Isoyan, Q. Leonard, M. Fisher, M. Green, J. Bisognano, P. F. Nealey, and F. Cerrina, Nucl. Instrum. Methods Phys. Res. A 582, 254 (2007).

${ }^{10}$ A. Ritucci, A. Reale, P. Zuppella, L. Reale, P. Tucceri, G. Tomassetti, P. Bettotti, and L. Pavesi, J. Appl. Phys. 102, 034313 (2007).

${ }^{11}$ M. Goldstein, S. H. Lee, Y. A. Shroff, P. J. Silverman, D. Williams, H. Park, M. A. Piestrup, and R. H. Pantell, Proceedings of the 2005 FEL Conference, 21-26 August 2005, Stanford, CA (2005), p. 422.

${ }^{12}$ K. Petrillo et al., J. Vac. Sci. Technol. B 25, 2490 (2007).

${ }^{13}$ R. Gronheid, H. H. Solak, Y. Ekinci, A. Jouve, and F. Van Roey, Microelectron. Eng. 67-68, 1103 (2006).

${ }^{14}$ B. Paivanranta, A. Langner, E. Kirk, C. David, and Y. Ekinci, Nanotechnology 22, 375302 (2011).

${ }^{15}$ H. H. Solak and Y. Ekinci, J. Vac. Sci. Technol. B 23, 2705 (2005).

${ }^{16} \mathrm{~K}$. Patroski, in Progress in Optics, edited by E. Wolf (North-Holland, Amsterdam, 1989), Vol. 27, pp. 1-108.

${ }^{17}$ H. H. Solak, C. Dais, and F. Clube, Opt. Express 19, 10686 (2011).

${ }^{18}$ B. L. Henke, E. M. Gullikson, and J. C. Davis, At. Data Nucl. Data Tables 54, 181 (1993).

${ }^{19}$ L. Wang, H. H. Solak, and Y. Ekinci (unpublished).

${ }^{20}$ Y. Ekinci, H. H. Solak, C. Padeste, J. Gobrecht, M. P. Stoykovich, and P. F. Nealey, Microelectron. Eng. 84, 700 (2007).

${ }^{21}$ A. E. Grigorescu and C. W. Hagen, Nanotechnology 20, 292001 (2009).

${ }^{22}$ J. W. Thackeray, M. Wagner, S. J. Kang, and J. Biafore, J. Photopolym. Sci. Technol. 23, 631 (2010). 